\title{
openheart Impact of a single non-sex-related stroke risk factor on atrial fibrillation and oral anticoagulant outcomes: a systematic review and meta-analysis
}

\author{
Maxim Grymonprez (D , ${ }^{1}$ Stephane Steurbaut, ${ }^{2}$ An De Sutter, ${ }^{3}$ Lies Lahousse ${ }^{1,4}$
}

To cite: Grymonprez M, Steurbaut S, De Sutter A, et al. Impact of a single non-sexrelated stroke risk factor on atrial fibrillation and oral anticoagulant outcomes: a systematic review and metaanalysis. Open Heart 2020;7:e001465. doi:10.1136/ openhrt-2020-001465

Received 30 September 2020 Revised 9 November 2020 Accepted 7 December 2020
Check for updates

(c) Author(s) (or their employer(s)) 2020. Re-use permitted under CC BY-NC. No commercial re-use. See rights and permissions. Published by BMJ.

${ }^{1}$ Department of Bioanalysis, Pharmaceutical Care Unit, Ghent University, Ghent, Belgium ${ }^{2}$ Centre for Pharmaceutical Research, Research Group of Clinical Pharmacology and Clinical Pharmacy, Vrije Universiteit Brussel, Jette, Belgium

${ }^{3}$ Department of Public Health and Primary Care, Faculty of Medicine and Health Sciences, Ghent University, Ghent, Belgium ${ }^{4}$ Department of Epidemiology, Erasmus Medical Center, Rotterdam, The Netherlands

Correspondence to Maxim Grymonprez; maxim. grymonprez@ugent.be

\section{ABSTRACT}

Aims Oral anticoagulants (OACs) are crucial for treating atrial fibrillation (AF) patients at high thromboembolic risk. However, in AF patients at intermediate thromboembolic risk with a single non-sex-related stroke risk factor (CHA ${ }_{2} \mathrm{DS}_{2}$-VASc score 1 in men, 2 in women), guidelines advise to consider starting anticoagulation, which may result in $\mathrm{OAC}$ non-initiation due to underestimation of the thromboembolic risk of a single stroke risk factor and overestimation of the OAC-related bleeding risk. A critical appraisal of the role of OACs and the benefit-risk profile of non-vitamin K antagonist oral anticoagulants (NOACs) compared with vitamin $\mathrm{K}$ antagonists (VKAs) in this patient subgroup are needed.

Methods and results This systematic review provides an overview of literature on the effectiveness and safety of OACs in AF patients with a single non-sex-related stroke risk factor after searching Medline and Embase. Differences between individual stroke risk factors regarding the ischaemic stroke risk in non-anticoagulated AF patients are identified in a meta-analysis, demonstrating the highest increased risk in patients aged 65-74 years old or with diabetes mellitus, followed by heart failure, hypertension and vascular disease. Furthermore, meta-analysis results favour NOACs over VKAs, given their equal effectiveness and superior safety in AF patients at intermediate thromboembolic risk (HR 0.93, $95 \% \mathrm{Cl} 0.65$ to 1.34 for stroke or systemic embolism; HR $0.60,95 \% \mathrm{Cl} 0.45$ to 0.80 for major bleeding; HR 0.48 , $95 \% \mathrm{Cl} 0.14$ to 1.59 for intracranial bleeding; HR 0.58 , $95 \% \mathrm{Cl} 0.47$ to 0.71 for mortality).

Conclusion Our systematic review with meta-analysis favours the use of anticoagulation in AF patients with a single non-sex-related stroke risk factor, especially when age $\geq 65$ years or diabetes mellitus is present, with a preference for NOACs over VKAs.

\section{INTRODUCTION}

Atrial fibrillation (AF) is the most common cardiac arrhythmia worldwide and is associated with a fivefold increased risk of stroke or systemic embolism (stroke/SE). ${ }^{1}$ Oral anticoagulants (OACs) are the cornerstone of AF treatment to reduce the thromboembolic risk. $^{2}$ Large randomised controlled trials

\section{Key questions}

What is already known about this subject?

- Guidelines advise to consider starting oral anticoagulants (OACs) in atrial fibrillation (AF) patients at intermediate thromboembolic risk with a single non-sex-related stroke risk factor $\left(\mathrm{CHA}_{2} \mathrm{DS}_{2}-\mathrm{VASc}\right.$ score 1 in men, 2 in women). Underestimation of the thromboembolic risk and overestimation of the OAC-related bleeding risk may result in OAC non-initiation.

What does this study add?

- In non-anticoagulated AF patients with a single nonsex-related stroke risk factor, not all risk factors were associated with a similar increase in ischaemic stroke risk, as the risk increased most significantly in patients aged between 65 and 74 years old or with diabetes mellitus, followed by congestive heart failure, hypertension or vascular disease.

- Importantly, in line with results in AF patients at high thromboembolic risk, this is the first meta-analysis demonstrating that non-vitamin $\mathrm{K}$ antagonist ora anticoagulants (NOACS) may be preferred over vitamin $\mathrm{K}$ antagonists in AF patients at intermediate thromboembolic risk, given the similar effectiveness and superior safety.

How might this impact on clinical practice?

- In absence of absolute contra-indications for anticoagulation, every AF patient with a single non-sexrelated stroke risk factor appears to benefit from treatment with a NOAC, after careful evaluation of modifiable bleeding risk factors.

(RCTs) comparing non-vitamin $\mathrm{K}$ antagonist oral anticoagulants (NOACs) with vitamin $\mathrm{K}$ antagonists (VKAs) in patients with nonvalvular AF, have shown that NOACs are efficacious and safe alternatives to VKAs. ${ }^{3-6}$ International guidelines ${ }^{7}$ have expressed a preference for NOACs over VKAs in AF patients without clear contra-indications for OACs. ${ }^{7-10}$ They recommend initiation of an OAC in the presence of $\geq 2$ non-sex-related stroke risk factors (high thromboembolic risk), using the 
$\mathrm{CHA}_{2} \mathrm{DS}_{2}$-VASc score (score of $\geq 2$ in men, $\geq 3$ in women, based on congestive heart failure, hypertension, age $\geq 75$ years (2 points), diabetes mellitus, stroke/SE/transient ischaemic attack (TIA) (2 points), vascular disease, age 65-74 years and female sex). In AF patients without a nonsex-related stroke risk factor (low thromboembolic risk: $\mathrm{CHA}_{2} \mathrm{DS}_{2}$-VASc score of 0 in men, 1 in women), OACs should be omitted. However, in AF patients at intermediate thromboembolic risk with only one non-sex-related stroke risk factor $\left(\mathrm{CHA}_{2} \mathrm{DS}_{2}-\mathrm{VASc}\right.$ score of 1 in men and 2 in women), discrepancies are still present. The European $^{810}$ and American ${ }^{9}$ guidelines recommend that OACs should be considered in AF patients with a single non-sex-related stroke risk factor, whereas the Canadian guidelines $^{7}$ recommend $\mathrm{OAC}$ initiation in these patients (instead of 'consider an OAC'). Due to the ambiguous advice of guidelines 'to consider anticoagulation', clinicians are left in uncertainty whether or not to start anticoagulation in AF patients with a single non-sex-related stroke risk factor.

In order for the clinician to decide whether anticoagulation is useful or not in patients at intermediate thromboembolic risk, three important aspects should be evaluated. First, does the presence of one stroke risk factor significantly increase the thromboembolic risk in non-anticoagulated AF patients? Second, does the reduction in the thromboembolic risk by starting an OAC in AF patients at intermediate thromboembolic risk outweigh the potential increase in the bleeding risk? Third, if a net clinical benefit for OAC initiation is present, do NOACs provide the same effectiveness and safety as VKAs in these AF patients? This systematic review provides an overview of the literature investigating these research questions. A meta-analysis on the impact of each individual non-sexrelated stroke risk factor on the risk of ischaemic stroke in non-anticoagulated AF patients will be performed to identify differences between risk factors, as well as a metaanalysis on the effectiveness and safety of NOACs versus VKAs in AF patients at intermediate thromboembolic risk.

\section{METHODS}

Using the Medline and Embase databases, a literature search was performed (see online supplemental etable 1). Longitudinal studies related to (1) the impact of each individual non-sex-related stroke risk factor according to the $\mathrm{CHA}_{2} \mathrm{DS}_{2}$-VASc score on the risk of ischaemic stroke in non-anticoagulated non-valvular AF patients as compared with $\mathrm{AF}$ patients with no stroke risk factors (first metaanalysis), (2) the effectiveness and safety of OACs versus no OACs in AF patients with one stroke risk factor and (3) the effectiveness and safety of NOACs versus VKAs in AF patients with a $\mathrm{CHA}_{2} \mathrm{DS}_{2}$-VASc score of 1 in men and 2 in women (second meta-analysis) during a mean/median follow-up of at least 6 months were included. If studies only reported results on the effectiveness and safety of NOACs versus VKAs in AF patients with a low $\mathrm{CHADS}_{2}$ score $(\leq 1)$ but not stratified according to $\mathrm{CHA}_{2} \mathrm{DS}_{2}$-VASc score, they were excluded for the second meta-analysis, but still included for the qualitative synthesis (systematic review). Studies regarding OAC use for non-AF indications (eg, venous thromboembolism) were excluded. Effectiveness and safety outcomes of interest were thromboembolism (ischaemic stroke or stroke/SE), all-cause mortality, major bleeding, intracranial bleeding and gastrointestinal bleeding. RCTs (post hoc analyses or original trial), longitudinal observational cohort studies and meta-analyses written in English were included for the qualitative synthesis, while cross-sectional studies, reviews, case reports, editorials or conference proceedings were not considered. For both meta-analyses, only (post hoc analyses of) RCTs and longitudinal observational cohort studies were included. No restriction of publication date was used.

Up to 1 November 2020, 6435 articles were identified. Additional articles of interest were selected by screening the reference list of identified studies. After screening title and abstract, 36 articles were selected. After reading the full-text, 13 articles were selected for the qualitative synthesis, 3 for the first meta-analysis (all observational studies) and 3 for the second meta-analysis (1 post hoc analysis of an RCT, 2 observational studies) (figure 1). The study design, patient characteristics and outcome measures of included studies are displayed in tables (online supplemental etable 2-4).

Both meta-analyses were performed using the metafor package in R (R V.3.6.1 with RStudio V.1.2.5001) with a random effects model and inverse-variance weighting, by pooling results based on the logarithm of the adjusted hazard ratios (HRs) and standard error. Data on study characteristics (design, setting and duration), baseline characteristics of included subjects (total number and mean/median age), comparison (eg, NOAC vs VKA) and the effectiveness and safety outcomes of interest (ischaemic stroke for the first meta-analysis; stroke/SE, major bleeding, intracranial bleeding and all-cause mortality for the second meta-analysis) were extracted from the original publications or supplemental materials. Effect sizes were presented as HR with 95\% CI) for the outcomes of interest when comparing non-anticoagulated AF patients with one stroke risk factor to no stroke risk factors (first meta-analysis), and NOACs to VKAs in AF patients at intermediate thromboembolic risk $\left(\mathrm{CHA}_{2} \mathrm{DS}_{2}\right.$-VASc score 1 in men, 2 in women) (second meta-analysis). A two-sided $\mathrm{p}$ value of $<0.05$ was considered statistically significant. Forest plots were drafted using the forestplot package in R. Heterogeneity was tested using the $\mathrm{I}^{2}$ statistic, based on a restricted maximum-likelihood estimator.

The risk of bias of each study included in the metaanalyses was assessed using the quality assessment tool 'QUALSYST' from the 'Standard Quality Assessment Criteria for Evaluating Primary Research Papers from a Variety of Fields' (online supplemental etable 5,6). ${ }^{11}$ Fourteen items of each quantitative study were scored with this tool on the study and outcome levels depending 


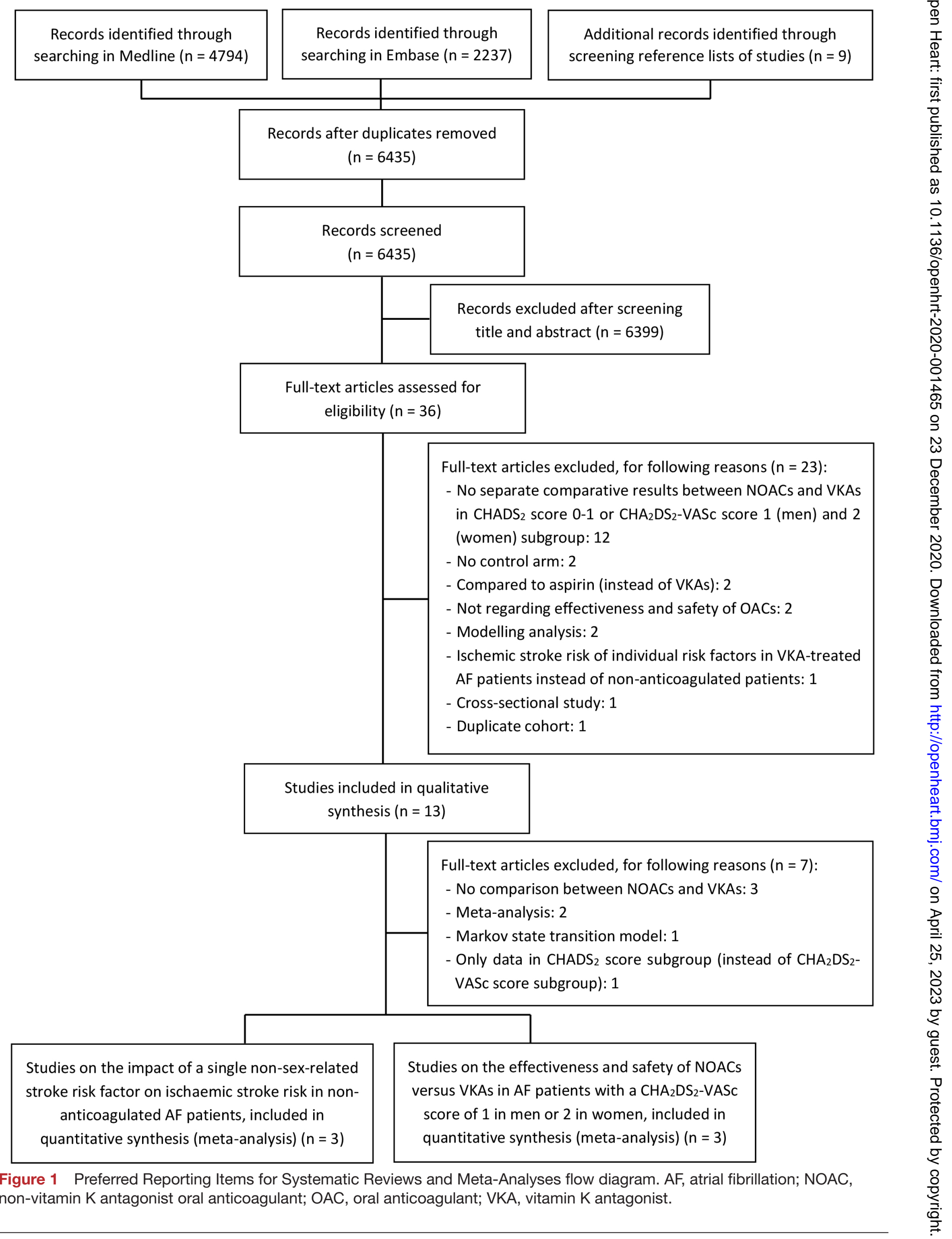


on the degree to which the specific criteria were met or reported ('yes' $=2$, 'partial' $=1$, 'no' $=0$, ' $\mathrm{n} / \mathrm{a}$ ' if not applicable to a particular study design). A percentage was calculated for each paper by dividing the total sum score obtained across rated items by the total possible score. Studies were included if scoring at least $80 \%$ on the quality assessment tool. The risk of publication bias at the outcome level for the studies included in the metaanalyses was assessed through funnel plot asymmetry and Egger's regression test. This work has been performed according to the Preferred Reporting Items for Systematic Reviews and Meta-Analyses (PRISMA) guidelines (PRISMA checklist included in supplemental materials, online supplemental etable 7).

\section{RESULTS}

\section{Impact of single non-sex-related stroke risk factor} Systematic review

Several studies have investigated the impact of each individual non-sex-related stroke risk factor on the risk of ischaemic stroke in non-anticoagulated AF patients (online supplemental etable 2). In a Danish cohort study by Olesen $e t a l$, an overall thromboembolic rate of 0.78 per 100 person-years (PY) was found in 6369 nonanticoagulated AF patients with no risk factors versus 2.01 per $100 \mathrm{PY}$ in 8203 patients with a single risk factor after 1 year of follow-up. ${ }^{12}$ Diabetes mellitus and age 65-74 years old were associated with the highest increase in the thromboembolic risk as compared with subjects without risk factors. Hypertension showed an intermediately increased thromboembolic risk, while vascular disease and congestive heart failure did not significantly increase the risk after 1 year. Similar results were seen after 5 and 10 years of follow-up, although the thromboembolic risk also significantly increased in patients with vascular disease or heart failure, which may indicate that longterm treatment is required until being beneficial.

Similarly, a Taiwanese cohort study by Chao et al demonstrated an annual ischaemic stroke rate of 2.75 and 2.55 per $100 \mathrm{PY}$ in 12935 male and 7900 female nonanticoagulated AF patients, respectively, with a single non-sex-related stroke risk factor after a mean follow-up of 5.2 years. ${ }^{13}$ As compared with $\mathrm{AF}$ patients without a stroke risk factor, the highest increase in ischaemic stroke risk was observed in patients aged 65-74 years old and patients with diabetes mellitus, followed by congestive heart failure, hypertension and vascular disease.

Moreover, in another Taiwanese cohort study by Hung et alstratifying non-anticoagulated $\mathrm{AF}$ patients by age, the annual ischaemic stroke rate in 20-49 and 50-64 years old patients without any non-sex-related stroke risk factor ( $\mathrm{n}=3674$ and 4301 ) was 0.63 and 1.96 per $100 \mathrm{PY}$, respectively, whereas 1.33 and 2.90 per $100 \mathrm{PY}$ in patients with one non-sex-related stroke risk factor aged 20-49 and $50-64$ years old, respectively ( $\mathrm{n}=1852$ and 4561 ) and 3.60 per $100 \mathrm{PY}$ in patients $65-74$ years old $(\mathrm{n}=5422) .{ }^{14}$ The highest increase in ischaemic stroke risk was observed in younger AF patients ( $<50$ years old) with heart failure or diabetes mellitus as compared with age-matched $\mathrm{AF}$ patients without risk factors. Hypertension and vascular disease also significantly increased the risk, but less than heart failure or diabetes mellitus. Likewise, in AF patients aged 50-64 years old, diabetes mellitus was the most important risk factor, followed by congestive heart failure and hypertension, but vascular disease was not associated with a significantly increased ischaemic stroke risk. Finally, age 65-74 years old as a single stroke risk factor also significantly increased the risk of ischaemic stroke as compared with younger $\mathrm{AF}$ patients without stroke risk factors.

\section{Meta-analysis}

Results of the three above-mentioned longitudinal observational cohort studies ${ }^{12-14}$ were pooled in a meta-analysis. All included studies scored $>80 \%$ on the quality assessment tool 'QUALSYST'11 (online supplemental etable $5)$. As compared with non-anticoagulated AF patients without stroke risk factors, the presence of any non-sexrelated stroke risk factor $\left(\mathrm{CHA}_{2} \mathrm{DS}_{2}\right.$-VASc score 1 in men, 2 in women) was associated with a more than doubled risk of ischaemic stroke (HR 2.03, 95\% CI 1.65 to 2.51, $\mathrm{I}^{2}$ $93.3 \%$ ) (figure 2). The overall pooled ischaemic stroke risk was most significantly increased in AF patients aged 65-74 years old (HR 3.24, 95\% CI 2.25 to 4.69 , I $97.6 \%$ ) and patients with diabetes mellitus (HR 2.51, 95\% CI 2.21 to $2.84, \mathrm{I}^{2} 26.9 \%$ ) as compared with $\mathrm{AF}$ patients without stroke risk factors, followed by congestive heart failure (HR $1.98,95 \%$ CI 1.58 to 2.50, I $^{2} 83.9 \%$ ) and hypertension (HR 1.74, 95\% CI 1.50 to 2.02 , I $\mathrm{I}^{2} 71.9 \%$ ). Vascular disease was associated with the smallest but still significantly increased ischaemic stroke risk (HR 1.56, 95\% CI 1.14 to $2.12, \mathrm{I}^{2} 72.4 \%$ ).

No publication bias was suspected, although the interpretation may have not been reliable, as less than 10 studies were included in the meta-analysis (online supplemental efigure 3). The overall substantial heterogeneity observed for all risk factors (except for diabetes mellitus) was likely caused by pooling results of the study by Hung et $a l^{14}$ that stratified the cohort by age. In this study, $27.9 \%$ and $44.7 \%$ of patients were 20-49 and 50-64 years old, respectively. In the study of Olesen $e t a l,{ }^{12}$ only $20.5 \%$ of patients were $<65$ years old, while in the study of Chao $e t a l,{ }^{13}$ the mean age was 59.1 years \pm 11.3 and 59.1 years \pm 10.2 for male and female AF patients, respectively, which may indicate that these studies included relatively older AF patients than the study of Hung et al. As a sensitivity analysis, results of the 20-49 and 50-64 years old patients included in the study of Hung et $a l^{14}$ were first combined (as one group $<65$ years old), and then subsequently pooled with the results of the other two included studies. Similar trends were observed, though substantial heterogeneity was no longer present (except for hypertension) (online supplemental efigure 1). 


\section{Overall}

Chao et al. 2015 (male)

Chao et al. 2015 (female)

Hung et al. 2016 (20-49y)

Hung et al. 2016 (50-64y)

Summary statistic (RE model)

Heterogeneity: $I^{2}=93.3 \%, Q=55.82, p(Q)<0.001$

1.1 Congestive heart failure

Olesen et al. 2011

Chao et al. 2015 (male)

Chao et al. 2015 (female)

Hung et al. 2016 (20-49y)

Hung et al. 2016 (50-64y)

Summary statistic (RE model)

Heterogeneity: $I^{2}=83.9 \%, Q=23.1, p(Q)<0.001$

\subsection{Hypertension}

Olesen et al. 2011

Chao et al. 2015 (male)

Chao et al. 2015 (female)

Hung et al. 2016 (20-49y)

Hung et al. 2016 (50-64y)

Summary statistic (RE model)

Heterogeneity: $I^{2}=71.9 \%, Q=16.2, p(Q)=0.003$

\subsection{Age 65-74 years}

Olesen et al. 2011

Chao et al. 2015 (male)

Chao et al. 2015 (female)

Hung et al. 2016 (65-74y vs 50-64y)

Hung et al. 2016 (65-74y vs 20-49y)

Summary statistic (RE model)

Heterogeneity: $I^{2}=97.6 \%, Q=182.3, p(Q)<0.001$

\subsection{Diabetes mellitus}

Olesen et al. 2011

Chao et al. 2015 (male)

Chao et al. 2015 (female)

Hung et al. 2016 (20-49y)

Hung et al. 2016 (50-64y)

Summary statistic (RE model)

Heterogeneity: $I^{2}=26.9 \%, Q=5.80, p(Q)=0.21$

$\begin{array}{ll}\text { NR } & 6369 \\ 4960 & 9416 \\ 2641 & 6390 \\ 5422 & 3674 \\ 5422 & 4301 \\ \text { NA } & \mathbf{3 0 1 5 0}\end{array}$

$\begin{array}{ll}\text { NR } & 6369 \\ 4075 & 9416 \\ 2756 & 6390 \\ 705 & 3674 \\ 2230 & 4301 \\ \text { NA } & \mathbf{3 0 1 5 0}\end{array}$

$2.92[1.72-4.96] \quad 6.3 \%$

$1.95[1.73-2.19] \quad 26.9 \%$

$1.71[1.48-1.98] \quad 24.5 \%$

$1.59[1.21-2.09] \quad 15.4 \%$

$1.46[1.31-1.64] \quad 26.9 \%$

$1.74[1.50-2.02] 100.0 \%$

\section{$2.39[2.18-2.60] \quad 26.1 \%$ \\ $2.12[1.76-2.55] 22.5 \%$ \\ $1.51[1.37-1.66] \quad 25.9 \%$ \\ $2.03[1.65-2.51] 100.0 \%$}

$2.40[0.58-9.98] \quad 2.4 \%$

$2.06[1.79-2.37] \quad 25.6 \%$

1.98 [1.67-2.35] $24.5 \%$

$1.46[1.27-1.68] 25.6 \%$

$1.98[1.58-2.50] 100.0 \%$

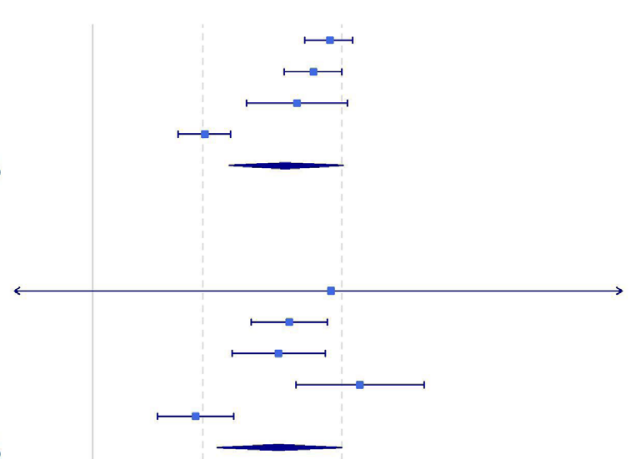

$3.54 \quad[2.33-5.38] \quad 16.7 \%$

$3.09[2.79-3.41] \quad 20.9 \%$

3.03 [2.68-3.43] $20.8 \%$

$1.90[1.73-2.08] \quad 21.0 \%$

$5.87[5.10-6.76] \quad 20.6 \%$

$3.24[2.25-4.69] 100.0 \%$

$\begin{array}{lllll}\text { NR } & 6369 & 4.77 & {[2.01-11.31]} & 2.0 \% \\ 1097 & 9416 & 2.66 & {[2.23-3.16]} & 31.1 \% \\ 701 & 6390 & 2.66 & {[2.16-3.27]} & 24.7 \% \\ 224 & 3674 & 2.53[1.73-3.70] & 9.5 \% \\ 679 & 4301 & 2.17 & {[1.84-2.56]} & 32.7 \% \\ \text { NA } & \mathbf{3 0 1 5 0} & \mathbf{2 . 5 1}[\mathbf{2 . 2 1 - 2 . 8 4}] & \mathbf{1 0 0 . 0} \%\end{array}$

$4.77[2.01-11.31] 2.0 \%$

$2.66[2.23-3.16] \quad 31.1 \%$

$2.53[1.73-3.70] \quad 9.5 \%$

$2.51[2.21-2.84] 100.0 \%$

\subsection{Vascular disease}

Olesen et al. 2011

Chao et al. 2015 (male)

Chao et al. 2015 (female)

Hung et al. 2016 (20-49y)

Hung et al. 2016 (50-64y)

Summary statistic (RE model)

Heterogeneity: $I^{2}=72.4 \%, Q=14.89, p(Q)=0.005$ clinical benefit for the use of warfarin in $2262 \mathrm{AF}$ patients with a single stroke risk factor as compared with no OAC use $(n=20103)$ after both 1 and 5 years of follow-up (based on the risk of stroke, major bleeding and myocardial infarction, weighted for the risk of death following an event). ${ }^{15}$ In contrast, a neutral net clinical benefit was observed in 1563 warfarin-treated AF patients without stroke risk factors as compared with no OAC use $(\mathrm{n}=23$ 219). Moreover, in the Swedish cohort study by Friberg et al, warfarin use was associated with a positive net clinical benefit (based on the avoided ischaemic strokes with without stroke risk factors. ${ }^{12}$ Likewise, another Danish cohort study by Lip et al also demonstrated a positive net 
warfarin minus the excess intracranial haemorrhages with warfarin) in AF subjects with a $\mathrm{CHA}_{2} \mathrm{DS}_{2}$-VASc of 1 , but not in subjects with a score of $0 .{ }^{16}$ Finally, in the French prospective cohort study by Fauchier et al, VKA use $(n=600)$ in AF patients with a single non-sex-related stroke risk factor was associated with a significantly lower risk of stroke/SE/death as compared with no OAC use $(n=499)$, while this was not the case in subjects without stroke risk factors. ${ }^{17}$

\section{Effectiveness and safety of NOACs versus VKAs \\ Systematic review}

Two secondary analyses of RCTs have been performed in AF patients at intermediate thromboembolic risk (online supplemental etable 4 ). In a post hoc analysis of the RE-LY trial by Oldgren et al, the use of dabigatran $150 \mathrm{mg}$ two times per day was associated with significantly lower stroke/SE, major bleeding, intracranial bleeding and all-cause mortality risks as compared with warfarin in AF patients with a $\mathrm{CHADS}_{2}$ score of $0-1 \quad(n=5323)$. In contrast, dabigatran $110 \mathrm{mg}$ two times per day had similar stroke/SE and all-cause mortality risks, but significantly lower major bleeding and intracranial bleeding risks (no separate data in $\mathrm{CHA}_{2} \mathrm{DS}_{2}$-VASc score subgroups). ${ }^{18}$ Similarly, in a secondary analysis of the ARISTOTLE trial by Lopes $e$ t $a l$, apixaban was associated with a similar stroke/SE and all-cause mortality risk, but a significantly lower major bleeding and intracranial bleeding risk as compared with warfarin in AF patients with a $\mathrm{CHADS}_{2}$ score of 1 ( $n=3100$ and 3083 , respectively) ${ }^{19}$ However, in AF patients with a $\mathrm{CHA}_{2} \mathrm{DS}_{2}$-VASc score of $1 \quad(\mathrm{n}=1604)$, similar risks for all outcomes were observed when comparing apixaban to warfarin, although these results were largely underpowered to detect small differences. After pooling of results in AF patients with a $\mathrm{CHADS}_{2}$ score of $0-1$ from the RE-LY and ARISTOTLE trial $(n=11$ 958), the meta-analysis by Lega et al illustrated a similar stroke/SE risk, but significantly lower major bleeding risk when comparing NOACs to warfarin. ${ }^{20}$

Besides these limited yet exploratory randomised data, results from observational studies were largely consistent (online supplemental etable 4). A Danish cohort study by Lip et al, including AF patients with one nonsex-related stroke risk factor, observed similar stroke/SE risks and significantly lower all-cause mortality risks for each individual standard dose NOAC (3272 dabigatran, 1604 rivaroxaban and 1470 apixaban users) as compared with warfarin $(n=7674)$ after 1 and 2.5 years of follow-up (except for a similar mortality risk for rivaroxaban after 2.5 years of follow-up). ${ }^{21}$ Only dabigatran and apixaban were associated with a significantly lower major bleeding risk as compared with warfarin, whereas rivaroxaban with a similar risk. Similarly, in an observational cohort study of Coleman et al, the use of rivaroxaban $20 \mathrm{mg}$ once daily in 3319 AF patients with a single non-sex-related stroke risk factor was associated with a significantly lower stroke/SE risk, and similar major bleeding, intracranial bleeding and gastrointestinal bleeding risks as compared with warfarin $(\mathrm{n}=3319)$ after 1 and 2 years of follow-up. ${ }^{22}$

\section{Meta-analysis}

Results from one randomised study (post hoc analysis of the ARISTOTLE trial) ${ }^{19}$ and two observational cohort studies $^{2122}$ were pooled in a meta-analysis (figure 3). Results of the RE-LY trial were not included, as no data were provided in the $\mathrm{CHA}_{2} \mathrm{DS}_{2}$-VASc score subgroups. ${ }^{18}$ All included studies scored $>80 \%$ on the quality assessment tool 'QUALSYST' ${ }^{11}$ (online supplemental etable 6).

Overall, NOACs were associated with a similar stroke/ SE (HR 0.93, 95\% CI 0.65 to $1.34, \mathrm{I}^{2} 29.2 \%$ ) and intracranial bleeding risk (HR $0.48,95 \%$ CI 0.14 to $1.59, \mathrm{I}^{2}$ $0.00 \%$ ), but a significantly lower major bleeding (HR 0.60, $95 \%$ CI 0.45 to $0.80, \mathrm{I}^{2} 21.4 \%$ ) and all-cause mortality risk (HR $0.58,95 \%$ CI 0.47 to $0.71, \mathrm{I}^{2} 0.00 \%$ ) as compared with warfarin. No substantial heterogeneity nor publication bias (online supplemental efigure 4) was observed. Given the low heterogeneity, an additional fixed effects model was performed, which rendered consistent results (online supplemental efigure 2).

\section{DISCUSSION}

The thromboembolic risk associated with AF substantially increases as more stroke risk factors are present in one patient. ${ }^{181923-25}$ Indeed, the ischaemic stroke rate in nonanticoagulated AF patients with a $\mathrm{CHA}_{2} \mathrm{DS}_{2}$-VASc score of 0,1 and 2 was $0.68,1.61$ and 2.49 per $100 \mathrm{PY}$, respectively, in a meta-analysis of 10 studies. $^{25}$ Intriguingly, in nonanticoagulated AF patients with a single non-sex-related stroke risk factor, not all risk factors were associated with a similar increase in ischaemic stroke risk. In our metaanalysis, the risk of ischaemic stroke in non-anticoagulated AF patients increased $224 \%$ and $151 \%$ for patients aged between 65 and 74 years old or with diabetes mellitus, respectively, as compared with $\mathrm{AF}$ patients without risk factors, followed by a 98\%, $74 \%$ and $56 \%$ significantly increased risk associated with congestive heart failure, hypertension or vascular disease, respectively (figure 2).

Furthermore, this systematic review highlights the beneficial role of OAC initiation over non-initiation in AF patients with a single non-sex-related stroke risk factor. The treatment threshold by which the OACinduced reduction in ischaemic stroke risk outweighs the increased bleeding risk, was estimated to be an annual ischaemic stroke risk of $>1.7 \%$ /year for warfarin and $>0.9 \%$ /year for NOACs in a Markov state transition decision model by Eckman et al, given the lower intracranial bleeding risk of NOACs. ${ }^{26}$ In AF patients at low thromboembolic risk, the thromboembolic event rate varied from 0.63 per $100 \mathrm{PY}^{14}$ in $\mathrm{AF}$ patients $<50$ years old to 0.78 per $100 \mathrm{PY}^{12}$ in $\mathrm{AF}$ patients $<65$ years old, which is below the estimated treatment threshold. ${ }^{12-14}$ OAC use in these AF patients did not significantly reduce the thromboembolic risk as compared with no OAC use, resulting in a neutral net clinical benefit. ${ }^{12}$ 15-17 These findings 
Author 1. Stroke/SE

Lip et al. 2017 (Obs: dabi 150) Coleman et al. 2019 (Obs: riva 20)

Lip et al. 2017 (Obs: riva 20)

Lopes et al. 2012 (RCT: api 5/2.5)

Lip et al. 2017 (Obs: api 5)

Summary statistic (RE model)

Heterogeneity: $\left.\right|^{2}=29.2 \%, Q=6.08, p(Q)=0.19$

n (NOAC) n (VKA) HR $95 \% \mathrm{Cl}$ Weight

$\begin{array}{lllll}3272 & 7674 & 0.81 & {[0.49-1.34]} & 29.7 \% \\ 3319 & 3319 & 0.41 & {[0.17-0.98]} & 13.9 \% \\ 1604 & 7674 & 1.46 & {[0.79-2.70]} & 23.2 \% \\ 802 & 802 & 1.18 & {[0.46-2.89]} & 13.3 \% \\ 1470 & 7674 & 1.01 & {[0.51-2.01]} & 19.9 \% \\ \mathbf{1 0 4 6 7} & \mathbf{2 7 1 4 3} & \mathbf{0 . 9 3} & {[\mathbf{0 . 6 5 - 1 . 3 4 ]}} & \mathbf{1 0 0 . 0 \%}\end{array}$

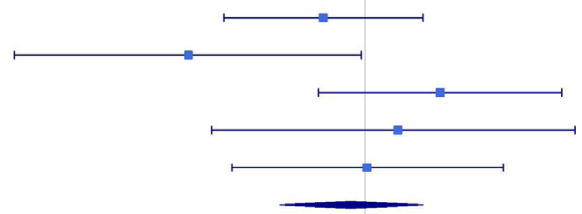

\section{Major bleeding}

Lip et al. 2017 (Obs: dabi 150)

Coleman et al. 2019 (Obs: riva 20)

Lip et al. 2017 (Obs: riva 20)

Lopes et al. 2012 (RCT: api 5/2.5)

Lip et al. 2017 (Obs: api 5)

Summary statistic (RE model)

Heterogeneity: $I^{2}=21.4 \%, Q=5.14, p(Q)=0.27$

$\begin{array}{lllll}3272 & 7674 & 0.48 & {[0.30-0.77]} & 27.3 \% \\ 3319 & 3319 & 0.74 & {[0.44-1.26]} & 22.9 \% \\ 1604 & 7674 & 0.84 & {[0.49-1.44]} & 22.5 \% \\ 802 & 802 & 0.65 & {[0.31-1.37]} & 13.3 \% \\ 1470 & 7674 & 0.35 & {[0.17-0.72]} & 14.0 \% \\ \mathbf{1 0 4 6 7} & \mathbf{2 7 1 4 3} & \mathbf{0 . 6 0} & {[\mathbf{0 . 4 5}-\mathbf{0 . 8 0}]} & \mathbf{1 0 0 . 0 \%}\end{array}$

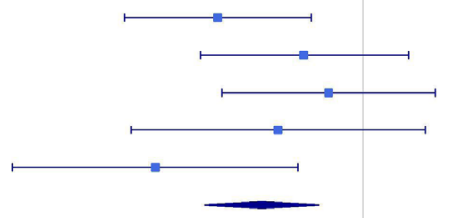

3. Intracranial bleeding

Coleman et al. 2019 (Obs: riva 20)

Lopes et al. 2012 (RCT: api 5/2.5)

Summary statistic (RE model)

$\begin{array}{lllll}3319 & 3319 & 0.33 & {[0.03-3.17]} & 28.4 \% \\ 802 & 802 & 0.55 & {[0.13-2.29]} & 71.6 \% \\ \mathbf{4 1 2 1} & \mathbf{4 1 2 1} & \mathbf{0 . 4 8} & {[\mathbf{0 . 1 4 - 1 . 5 9}]} & \mathbf{1 0 0 . 0 \%}\end{array}$

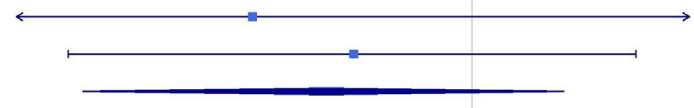

Heterogeneity: $I^{2}=0.00 \%, Q=0.14, p(Q)=0.71$

\section{All-cause mortality}

Lip et al. 2017 (Obs: dabi 150)

Lip et al. 2017 (Obs: riva 20)

Lopes et al. 2012 (RCT: api 5/2.5)

Lip et al. 2017 (Obs: api 5)

Summary statistic (RE model)

Heterogeneity: $\left.\right|^{2}=0.00 \%, Q=2.86, p(Q)=0.41$

$\begin{array}{lllll}3272 & 7674 & 0.59 & {[0.43-0.81]} & 41.4 \% \\ 1604 & 7674 & 0.52 & {[0.34-0.79]} & 23.8 \% \\ 802 & 802 & 0.82 & {[0.50-1.35]} & 16.8 \% \\ 1470 & 7674 & 0.47 & {[0.29-0.76]} & 18.0 \% \\ \mathbf{7 1 4 8} & \mathbf{2 3 8 2 4} & \mathbf{0 . 5 8} & {[\mathbf{0 . 4 7 - 0 . 7 1 ]}} & \mathbf{1 0 0 . 0 \%}\end{array}$

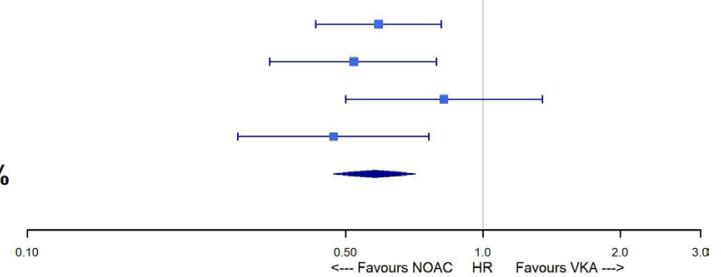

Figure 3 The risk of stroke/systemic embolism, major bleeding, intracranial bleeding and all-cause mortality of NOACs compared with warfarin in atrial fibrillation patients with a single non-sex-related stroke risk factor $\left(\mathrm{CHA}_{2} \mathrm{DS}_{2}-\mathrm{VASc}\right.$ score of 1 in men or in women). Api 5/2.5: apixaban $5 \mathrm{mg}$ (standard dose) or $2.5 \mathrm{mg}$ (reduced dose); Api 5: apixaban $5 \mathrm{mg}$ (standard dose); Dabi 150, dabigatran $150 \mathrm{mg}$ (standard dose); NOAC, non-vitamin K antagonist oral anticoagulant; Obs, longitudinal observational cohort study; RCT, randomised controlled trial (post hoc analysis); RE model: random effects model; Riva 20, rivaroxaban $20 \mathrm{mg}$ (standard dose); stroke/SE: stroke/systemic embolism; VKA, vitamin K antagonist.

confirm the recommendation of guidelines to not anticoagulate AF patients without any stroke risk factors. ${ }^{7-10}$ However, in AF patients with a single non-sex-related stroke risk factor, all individual stroke risk factors (except for vascular disease in one study ${ }^{12}$ ) were associated with ischaemic stroke rates above the treatment threshold for NOACs, with rates varying from 1.00 per $100 \mathrm{PY}^{14}$ in AF patients $<50$ years old with hypertension to 4.12 per 100 $\mathrm{PY}^{14}$ in AF patients aged 50-64 years with diabetes. ${ }^{12-14} 26$ Indeed, OAC use in these AF patients was associated with a positive net clinical benefit compared with no OAC use. $^{1215-17}$

Our meta-analysis results favour NOACs over VKAs in $\mathrm{AF}$ patients at intermediate thromboembolic risk, since these demonstrated similar stroke/SE and intracranial bleeding risks, but significantly lower major bleeding and all-cause mortality risks compared with warfarin (figure 3). However, lack of power limited the interpretability of the risk of intracranial bleeding. To the best of our knowledge, this is the first meta-analysis specifically investigating the effectiveness and safety of NOACs compared with VKAs in AF patients with a single non-sexrelated stroke risk factor according to the $\mathrm{CHA}_{2} \mathrm{DS}_{2}$-VASc score.

\section{Strengths and limitations}

The included studies had many strengths, such as the rigorous methodologies and well-defined cohorts of the (post hoc analyses of) RCTs, whereas the observational cohort studies included large groups of patients in a realworld setting with long follow-up durations. However, several limitations should be mentioned. First, data from randomised studies are scarce, since the pivotal phase III RCTs investigating the efficacy and safety of NOACs as compared with VKAs, mostly included AF patients at high thromboembolic risk. ${ }^{3-6}$ Indeed, the ROCKET-AF trial $^{4}$ for rivaroxaban and the ENGAGE-AF-TIMI 48 trial $^{6}$ for edoxaban only included patients at high thromboembolic risk with a $\mathrm{CHADS}_{2}$ score of $\geq 2$ (based on congestive heart failure, hypertension, age $\geq 75$ years, diabetes mellitus, stroke/SE/TIA (2 points)), so no randomised data for rivaroxaban or edoxaban in $\mathrm{AF}$ patients at 
intermediate thromboembolic risk were available. ${ }^{46}$ Only two phase III RCTs included patients at intermediate thromboembolic risk: the RE-LY trial $^{3}$ for dabigatran (inclusion of AF patients with at least one of the following stroke risk factors: prior stroke/TIA; congestive heart failure; age $\geq 75$ years and age $65-74$ years old in combination with diabetes mellitus, hypertension or coronary artery disease) and the ARISTOTLE trial ${ }^{5}$ for apixaban (inclusion of AF patients with a $\mathrm{CHADS}_{2}$ score of $\geq 1) .{ }^{35}$ In other words, AF patients $<65$ years old with heart failure included in the RE-LY trial and AF patients $<65$ years old with heart failure, hypertension or diabetes included in the ARISTOTLE trial would be categorised as intermediate thromboembolic risk, resulting in the limited available randomised data from the RE-LY ${ }^{18}$ and ARISTOTLE trial. ${ }^{19}$ Consequently, RCTs were underpowered for subgroups analyses. In line, as no phase III RCTs investigating the impact of individual non-sex-related stroke risk factors on the ischaemic stroke risk in nonanticoagulated AF patients were identified, we could only include observational studies for this meta-analysis. Second, most observational studies were retrospective in nature and were based on administrative healthcare data, mostly from Denmark or Taiwan, limiting the generalisability to other populations. When appraising the quality of studies using the 'QUALSYST' tool, the included observational studies $^{12-14} 2122$ lacked well-defined outcomes which were robust to measurement bias or were limited in their controlling for important confounders. Third, classification of patients at intermediate thromboembolic risk varied across studies due to the use of the CHADS $_{2}$ score instead of the $\mathrm{CHA}_{2} \mathrm{DS}_{2}$-VASc score. This is of major importance, as results in patients with a CHADS $_{2}$ score of 0 or 1 should not be extrapolated to AF patients with a $\mathrm{CHA}_{2} \mathrm{DS}_{2}$-VASc score of 1 in men or 2 in women, due to the poor ability of the CHADS $_{2}$ score to discriminate patients at low and intermediate stroke risk. ${ }^{8-101219}$ Theoretically, a patient with a $\mathrm{CHADS}_{2}$ score of 0 can have a $\mathrm{CHA}_{2} \mathrm{DS}_{2}$-VASc score of up to 3 in case of age 65-74 years old, vascular disease and female sex. Exemplary, in the ARISTOTLE trial, 7052 subjects were $65-74$ years old, 4500 subjects had vascular disease and 6416 subjects were women, so these large patient subgroups could have resulted in major differences between the CHADS $_{2}$ and the $\mathrm{CHA}_{2} \mathrm{DS}_{2}$-VASc score subgroups. ${ }^{19}$ To overcome this limitation, only studies reporting outcome data in patients according to the $\mathrm{CHA}_{2} \mathrm{DS}_{2}-\mathrm{VASc}$ score were included in the second meta-analysis. Fourth, in one study, ${ }^{19}$ female sex in absence of other stroke risk factors also put patients at intermediate thromboembolic risk $\left(\mathrm{CHA}_{2} \mathrm{DS}_{2}\right.$-VASc score 1$)$, while this was not the case for the other studies. ${ }^{2122}$ Fifth, due to the limited number of included studies, results of different (doses of) NOACs were pooled. Finally, in studies investigating the effectiveness and safety of NOACs versus VKAs, AF patients with a single stroke risk factor were pooled, but the proportion of each individual risk factor contributing to this group varied across studies. Most studies ${ }^{18} 1922$ included mainly patients with hypertension (proportion ranging from $58.8 \%^{18}$ to $77.3 \%^{19}$ ). However, one study ${ }^{21}$ especially included patients aged $65-74$ years old (59.3\% of cases), which may have influenced results, as thromboembolic and bleeding rates may have been higher in study cohorts especially including older AF patients.

\section{Recommendations and implications for clinical practice}

Based on the results of our meta-analysis and systematic review, an age of $\geq 65$ years old or diabetes mellitus, and to a lesser extent, congestive heart failure or hypertension, may warrant $\mathrm{OAC}$ initiation in $\mathrm{AF}$ patients. AF patients $<65$ years old with vascular disease and to a lesser extent $\mathrm{AF}$ patients $<50$ years old with a single risk factor, represent an 'indecisive area' for OAC initiation, for whom a thorough benefit-risk analysis with shared decision making is crucial. ${ }^{14}$

In these $\mathrm{AF}$ patients at intermediate thromboembolic risk, NOACs seem to be preferred over VKAs, given the non-inferior effectiveness and superior safety. Although no direct head-to-head comparisons have been performed, standard dose dabigatran ${ }^{18}$ and apixaban ${ }^{19}$ may be chosen for stroke prevention, based on preliminary randomised data. ${ }^{18} 19$ Observational data on standard dose rivaroxaban were reassuring as well, ${ }^{21}{ }^{22}$ while data on edoxaban are still lacking in this subgroup.

Regarding the implications for clinical practice, our results demonstrate that every AF patient with a single non-sex-related stroke risk factor without absolute contraindications for anticoagulation appears to benefit from NOAC treatment, after careful evaluation of modifiable bleeding risk factors, considering the estimated NOAC treatment threshold ${ }^{26}$ and the positive net clinical benefit for OAC initiation. ${ }^{12}{ }^{15-178} 10$ Physicians should be aware of the beneficial role of NOACs in this patient subgroup, instead of awaiting anticoagulation initiation until at least two risk factors are present.

\section{Research gaps}

Our systematic review identified considerable research gaps. First, more research is required on the effectiveness and safety of NOACs in young AF patients $<50$ years old at intermediate thromboembolic risk, as this subgroup represents an 'indecisive area' for OAC initiation. ${ }^{27}$ However, given the overall low number of identified studies in this systematic review, considerably more (randomised) data on NOACs versus VKAs are needed in this important population with a single non-sex-related stroke risk factor. Second, direct head-to-head comparisons between NOACs are still lacking, so currently no specific NOAC can be recommended for this patient subgroup. Third, the cost-effectiveness of systematically using NOACs in AF patients at intermediate thromboembolic risk should be assessed in future research.

\section{CONCLUSION}

In conclusion, our systematic review with meta-analysis favours the use of anticoagulation in $\mathrm{AF}$ patients with 
a single non-sex-related stroke risk factor. Especially AF patients $\geq 65$ years old or with diabetes mellitus may benefit from anticoagulation, as these risk factors were associated with the highest increase in ischaemic stroke risk in our meta-analysis, followed by congestive heart failure, hypertension and vascular disease. Importantly, in line with results in $\mathrm{AF}$ patients at high thromboembolic risk, this is the first meta-analysis demonstrating that NOACs may be preferred over VKAs in AF patients at intermediate thromboembolic risk, given the similar effectiveness and superior safety.

Twitter Maxim Grymonprez @Maxim Grymonprez

Contributors MG and LL contributed to the concept and design of the systematic review. MG performed the literature search, statistical analysis, interpretation and writing. SS, ADS and LL revised the systematic review critically. All authors contributed to the article and approved the submitted version.

Funding This work was supported by grants from the Fund for Scientific Research Flanders (FW0) [project number 11C0820N to M.G.].

Competing interests None declared.

Patient consent for publication Not required.

Provenance and peer review Not commissioned; externally peer reviewed.

Data availability statement All data relevant to the study are included in the article or uploaded as supplementary information.

Open access This is an open access article distributed in accordance with the Creative Commons Attribution Non Commercial (CC BY-NC 4.0) license, which permits others to distribute, remix, adapt, build upon this work non-commercially, and license their derivative works on different terms, provided the original work is properly cited, appropriate credit is given, any changes made indicated, and the use is non-commercial. See: http://creativecommons.org/licenses/by-nc/4.0/.

Correction notice This article has been corrected since it first published. The provenance and peer review statement has been included.

ORCID iD

Maxim Grymonprez http://orcid.org/0000-0002-0145-6486

\section{REFERENCES}

1 Wolf PA, Abbott RD, Kannel WB. Atrial fibrillation as an independent risk factor for stroke: the Framingham study. Stroke 1991;22:983-8.

2 Hart RG, Pearce LA, Aguilar MI. Meta-analysis: antithrombotic therapy to prevent stroke in patients who have nonvalvular atrial fibrillation. Ann Intern Med 2007;146:857-67.

3 Connolly SJ, Ezekowitz MD, Yusuf S, et al. Dabigatran versus warfarin in patients with atrial fibrillation. $N$ Engl J Med 2009;361:1139-51.

4 Patel MR, Mahaffey KW, Garg J, et al. Rivaroxaban versus warfarin in nonvalvular atrial fibrillation. N Engl J Med 2011;365:883-91.

5 Granger CB, Alexander JH, McMurray JJV, et al. Apixaban versus warfarin in patients with atrial fibrillation. N Engl J Med 2011;365:981-92.

6 Giugliano RP, Ruff CT, Braunwald E, et al. Edoxaban versus warfarin in patients with atrial fibrillation. N Engl J Med 2013;369:2093-104.

7 Andrade JG, Verma A, Mitchell LB, et al. 2018 focused update of the Canadian Cardiovascular Society guidelines for the management of atrial fibrillation. Can J Cardiol 2018;34:1371-92.

8 Steffel J, Verhamme P, Potpara TS, et al. The 2018 European Heart Rhythm Association Practical Guide on the use of non-vitamin K antagonist oral anticoagulants in patients with atrial fibrillation. Eur Heart J 2018;39:1330-93.
9 , January CT, Wann LS, et al, Writing Group Members. 2019 AHA ACC/HRS focused update of the 2014 AHA/ACC/HRS guideline for the management of patients with atrial fibrillation: a report of the American College of Cardiology/American Heart Association Task Force on Clinical Practice Guidelines and the Heart Rhythm Society. Heart Rhythm 2019;16:e66-93.

10 Hindricks G, Potpara T, Dagres N, et al. 2020 ESC guidelines for the diagnosis and management of atrial fibrillation developed in collaboration with the European Association for Cardio-Thoracic Surgery (EACTS). Eur Heart J 2020;20.

11 Kmet L, Lee R, Cook L. The quality assessment tool 'QUALSYST' from the "Standard Quality Assessment Criteria for Evaluating Primary Research Papers from a Variety of Fields", 2004. Available: https://www.ihe.ca/advanced-search/standard-quality-assessmentcriteria-for-evaluating-primary-research-papers-from-a-variety-offields [Accessed 1 August 2020].

12 Olesen JB, Lip GYH, Hansen ML, et al. Validation of risk stratification schemes for predicting stroke and thromboembolism in patients with atrial fibrillation: nationwide cohort study. BMJ 2011;342:d124-d.

13 Chao T-F, Liu C-J, Wang K-L, et al. Should atrial fibrillation patients with 1 additional risk factor of the CHA2DS2-VASc score (beyond sex) receive oral anticoagulation? J Am Coll Cardiol 2015;65:635-42.

14 Hung Y, Chao Tze-Fan, Liu Chia-Jen, Chao TF, Liu CJ, et al. Is an Oral Anticoagulant Necessary for Young Atrial Fibrillation Patients With a CHA ${ }_{2} \mathrm{DS}_{2}$-VASc Score of 1 (Men) or ${ }_{2}$ (Women)? J Am Heart Assoc 2016;5.

15 Lip GYH, Skjøth F, Nielsen PB, et al. Non-valvular atrial fibrillation patients with none or one additional risk factor of the CHA2DS2VASc score. A comprehensive net clinical benefit analysis for warfarin, aspirin, or no therapy. Thromb Haemost 2015;114:826-34.

16 Friberg L, Rosenqvist M, Lip GYH. Net clinical benefit of warfarin in patients with atrial fibrillation: a report from the Swedish atrial fibrillation cohort study. Circulation 2012;125:2298-307.

17 Fauchier L, Lecoq C, Clementy N, et al. Oral anticoagulation and the risk of stroke or death in patients with atrial fibrillation and one additional stroke risk factor: the Loire Valley atrial fibrillation project. Chest 2016;149:960-8.

18 Oldgren J, Alings M, Darius $\mathrm{H}$, et al. Risks for stroke, bleeding, and death in patients with atrial fibrillation receiving dabigatran or warfarin in relation to the CHADS2 score: a subgroup analysis of the RE-LY trial. Ann Intern Med 2011;155:660-7.

19 Lopes RD, Al-Khatib SM, Wallentin L, et al. Efficacy and safety of apixaban compared with warfarin according to patient risk of stroke and of bleeding in atrial fibrillation: a secondary analysis of a randomised controlled trial. Lancet 2012;380:1749-58.

20 Lega J-C, Bertoletti L, Gremillet C, et al. Consistency of safety and efficacy of new oral anticoagulants across subgroups of patients with atrial fibrillation. PLoS One 2014;9:e91398.

21 Lip GYH, Skjøth F, Nielsen PB, et al. Effectiveness and safety of standard-dose Nonvitamin $\mathrm{K}$ antagonist oral anticoagulants and warfarin among patients with atrial fibrillation with a single stroke risk factor: a nationwide cohort study. JAMA Cardiol 2017;2:872-81.

22 Coleman Cl, Turpie AGG, Bunz TJ, et al. Effectiveness and safety of rivaroxaban vs. warfarin in non-valvular atrial fibrillation patients with a non-sex-related CHA2DS2-VASc score of 1. Eur Heart $J$ Cardiovasc Pharmacother 2019;5:64-9.

23 Mentias A, Shantha G, Chaudhury P, et al. Assessment of outcomes of treatment with oral anticoagulants in patients with atrial fibrillation and multiple chronic conditions: a comparative effectiveness analysis. JAMA Netw Open 2018;1:e182870.

24 Hernandez I, Zhang Y, Saba S. Effectiveness and safety of direct ora anticoagulants and warfarin, stratified by stroke risk in patients with atrial fibrillation. Am J Cardiol 2018;122:69-75.

25 Joundi RA, Cipriano LE, Sposato LA, et al. Ischemic stroke risk in patients with atrial fibrillation and CHA2DS2-VASc score of 1 : systematic review and meta-analysis. Stroke 2016;47:1364-7.

26 Eckman MH, Singer DE, Rosand J, et al. Moving the tipping point: the decision to anticoagulate patients with atrial fibrillation. Circ Cardiovasc Qual Outcomes 2011;4:14-21.

27 Chao T-F, Lip GYH, Lin Y-J, et al. Age threshold for the use of nonvitamin $\mathrm{K}$ antagonist oral anticoagulants for stroke prevention in patients with atrial fibrillation: insights into the optimal assessment of age and incident comorbidities. Eur Heart J 2019;40:1504-14. 
Correction:Impact of a single non-sex-related stroke risk factor on atrial fibrillation and oral anticoagulant outcomes: a systematic review and meta-analysis

Grymonprez M, Steurbaut S, De Sutter A, et al. Impact of a single non-sex-related stroke risk factor on atrial fibrillation and oral anticoagulant outcomes: a systematic review and meta-analysis. Open Heart 2020;7:e001465. doi: 10.1136/openhrt-2020-001465.

This article has been corrected since it was first published. The provenance and peer review statement has been included.

Open access This is an open access article distributed in accordance with the Creative Commons Attribution Non Commercial (CC BY-NC 4.0) license, which permits others to distribute, remix, adapt, build upon this work non-commercially, and license their derivative works on different terms, provided the original work is properly cited, appropriate credit is given, any changes made indicated, and the use is non-commercial. See: http://creativecommons.org/licenses/by-nc/4.0/.

C Author(s) (or their employer(s)) 2021. Re-use permitted under CC BY-NC. No commercial re-use. See rights and permissions. Published by BMJ.

Open Heart 2021;8:e001465corr1. doi:10.1136/openhrt-2020-001465corr1

(A) Check for updates 